\title{
Specific meningoencephalitis in patients with transplanted kidney
}

\section{Mithat Tabakovic ${ }^{1 *}$, Senaid Trnacevic ${ }^{2}$ and Maida Dugonjic Taletovic ${ }^{3}$}

${ }^{1}$ University Clinical Center Tuzla, Bosnia and Herzegovina

${ }^{2}$ Medical School, University of Tuzla, Bosnia and Herzegovina

${ }^{3}$ Department of Nephrology, Clinic for Internal Diseases, University of Tuzla, Bosnia and Herzegovina

\section{Abstract}

We described a case of specific (tuberculous) encephalitis in a patient after kidney transplantation Immunosuppressive therapy, continuously required in post-tranplant period, may cause various complications, such as infections. Specific meningoencephalitis is an infection that is rarely diagnosed and more common in immunocompromised patients.

Case report: A 30-year-old man had kidney transplantation (kidney donor was his father). He previously was two years on chronic hemodialysis treatment because of end-stagerenal disease based on diabetic nephropathy. He has diabetes type 1 . The early post-transplant period duly passed with satisfactory clinical and laboratory parameters of renal function. Two months after transplantation, he presented with febrile condition, signs of septicemia and dehydration with significant neurological deficit and expressed meningeal signs. In cerebrospinal fluid we found lymphocytosis, elevated proteins and positive micobacterium tuberculosis antibodies (Hexagon method) and we suspected to specific etiology of meningitis. Performed computed tomography (CT) scan of the brain with contrast confirmed the expected finding.

Due to the poor prognosis of infections of the central nervous system (CNS) in immunocompromised patients, only prompt diagnosis can improve survival in this group of patients. The therapeutic protocol after kidney transplantation include the prophylactic use of antituberculous drug (Isoniazid $300 \mathrm{mg}$ ) during the 9 months.

\section{Introduction}

The history of clinical organ transplantation began in the 1960s with the introduction of azathioprine into therapy, which, along with steroids, was the basic immunosuppressive therapy for the next twenty years. In the $20^{\text {th }}$ century, kidney transplantation became the therapy of choice in the treatment of the terminal phase of chronic kidney disease.

Immunosuppressive therapy prevents rejection of the transplanted kidney, and the administration of this therapy primarily affects the success of the transplant. Immunosuppressive therapy is carried out continuously after the transplant procedure. Initial high doses are lowered during the first few weeks and maintenance therapy is continued. Immunosuppressive therapy can cause various posttransplant complications: infections, tumors, metabolic disorders, arterial hypertension, and other adverse events. One of the leading complications of immunosuppressive therapy is infection. A study by Peterson and colleagues in the early 1980s found that infections were the cause of death in $87 \%$ of kidney transplant patients [1]. During the first month after transplant surgery most infections are so called

\begin{abstract}
More Information
*Address for Correspondence: Mithat Tabakovic, University Clinical Center Tuzla, Bosnia and Herzegovina, Tel: 0038761178 534; Email: mithat.tabakovic@ukctuzla.ba; mithat.tabakovic@gmail.com
\end{abstract}

Submitted: 10 February 2020

Approved: 16 June 2020

Published: 17 June 2020

How to cite this article: Tabakovic M,

Trnacevic S, Taletovic MD. Specific

meningoencephalitis in patients with

transplanted kidney. J Clini Nephrol. 2020; 4: 024-026.

DOI: 10.29328/journal.jen.1001055

ORCiD: orcid.org/0000-0003-1017-4242

Copyright: (c) 2020 Tabakovic M, et al. This is an open access article distributed under the Creative Commons Attribution License, which permits unrestricted use, distribution, and reproduction in any medium, provided the original work is properly cited.

Keywords: Meningoencephalitis; Diabetes mellitus; Kidney transplantation; Antituberculous drug; Immunosuppression

\section{(D) Check for updates \\ OPEN ACCESS}

hospital infections (eg., bacteria of the genus Pseudomonas as agents for pneumonia; gram-positive bacteria that cause wound infection). Opportunistic infections usually occur from the first to six months after transplantation. According to the infectious agent, they can be divided into bacterial, viral (cytomegalovirus (CMV), Ebstein Barr virus (EBV), varicella zoster virus (VZV), hepatitis B or C virus), fungal or parasitic. The most significant viral infections are caused by cytomegalovirus and herpes virus. Infections caused by tuberculosis bacteria from different organs should also not be neglected. CNS infections are more common after kidney transplantation than in the general population: acute and chronic meningitis, brain abscess, and multifocal leukoencephalopathy. Listeria monocytogenes, criptococcus and mycobacterium tuberculosis are among the most common causes of meningitis. Specific meningoencephalitis is an inflammation of the meninges and brain parenchyma caused by mycobacterium tuberculosis, often undiagnosed and more common in immunocompromised patients (immunosuppressive therapy and HIV infection). Over a period of approximately six months the risk of infection decreases [2]. 


\section{Case report}

Female patient of 30 years of age, suffering from type 1 diabetes since the age of ten. As a result of diabetes nephropathy and terminal renal insufficiency, in November 2003, chronic hemodialysis treatment began. After two years of chronic dialysis treatment, the patient is hospitalized to prepare for kidney transplantation (live birth transplant), as a potential recipient, and the potential kidney donor was the father. Necessary findings and protocols for the recipient and donor, which included biochemical, microbiological, radiological and special specialist consultative findings, are made. In March 2005, a live family renal transplant was done. The postoperative course went well. The therapy protocol for the kidney recipient also includes standard immunosuppressive therapy, started the day before surgery. Body weight, fluid intake, diuresis, arterial blood pressure (TA), body temperature $\left(\mathrm{t}^{\circ} \mathrm{C}\right)$, blood cyclosporin level and glycemia were monitored daily. The creatinine level after transplantation is normalized and the blood cyclosporin level is within the recommended values. Ultrasound findings of the transplanted kidney were normal. The patient was discharged on the 18th posttransplant day with satisfactory clinical and laboratory parameters of renal function and in good general condition.

Two months after the transplant, the patient was hospitalized for fever $\left(38{ }^{\circ} \mathrm{C}\right)$, fever, headache, hearing loss, impaired consciousness, weakness, malaise, vomiting and oliguria. Physical examination determines the ability of the patient to follow the gaze, but verbal contact is not established. The impression is that it does not respond adequately to acoustic stimuli. The patient is febrile, tachypnoic, cachectic, poorly hydrated, immobile, giving the impression of a severe patient. The skin is pale with reddish maculae on the face and crosses on the lower legs. Heart and lung findings are normal, TA: 130/80 $\mathrm{mmHg}$, central pulse (CP: 90/min). The neurological status is dominated by the finding of impaired gross motor power at the right extremities, indicated meningeal signs, signs of central paresis $n$. facialisa right, bilateral nystagmus, but neck tightened with anteflexion. Accurate erythrocyte sedimentation (SE: 79/100), leukocytosis (L: 23), elevated $\mathrm{C}$ reactive protein (CRP: 61), signs of metabolic acidosis, elevated creatinine $239 \mu \mathrm{mol} / \mathrm{l}$, glycemia $26 \mathrm{mmol} / \mathrm{l}$ are recorded in laboratory findings. Urine positive glucose and ketone. The radiographic findings of the heart and lung were neat and the sputum on BK negative, as was the urine on Lowenstein. Ultrasound findings of the transplanted kidney show edema of the cortex, stressed pyramids, and index of resistance (RI 0.76). The ELISA test for EBV, CMV, herpes simplex virus (HSV) and VZV was IgM negative, IgG positive, and the CNS profile of IgG and IgM class from serum was negative. Computed tomography (CT) of the brain with contrast shows a wider ventricular system of the third and both lateral chambers, the initial type of hydrocephalus with discretely present periventricular hypodensity as seen with hydrocephalus accutation. CT findings do not rule out possible changes by type of meningitis. A lumbar puncture is performed in consultation with the infectologist. Liquor results in increased cell count up to 168 (lymphocytes), increased protein up to $3 \mathrm{~g} / \mathrm{l}$. Direct preparation of liquor on Micobacterium tuberculosis done five times was negative, and antibodies on Micobacterium tuberculosis in liquor (Hexagon), also made five times, were positive.

Acute meningitis is suspected. CT of the brain was done two more times, and the findings suggest a less pronounced paraventricular hypodensity. The electroencephalogram (EEG) speaks in favor of dysfunction of the frontal regions on both sides, and the finding of auditory evoked potentials (AEP) on both sides' remains without an evoked response. A diagnosis of specific meningoencephalitis is made on the basis of anamnestic data, clinical picture, physical examination, laboratory findings, CSF findings and CT brain. Quadruple antituberculosis (ATL) therapy is included which includes: rifadin, isoniazid, pyrazinamide, ethambutol. Two months after initiation of treatment, rifadin and isoniazid are continued for 4 months. The motor aphasia rehabilitation procedure included Logoped. Nephrologists, endocrinologists and neurologists were regularly examined. Satisfactory renal graft function (creatinine $108 \mu \mathrm{mol} / \mathrm{l}$ ) was achieved with adequate titration of cyclosporine. In a period of three months an improved general condition occurs with hemiparesis to the right, paresis $\mathrm{n}$. facialis right by cetral type, motor aphasia and impaired hearing.

\section{Discussion}

Diabetes mellitus is one of the most common causes of terminal wound failure. Kidney transplantation in these patients is the method of choice, although it is accompanied by numerous complications. These complications are reflected in an increased risk of infection as potentiated by immunosuppressive therapy, the recurrence of nephropathy in the transplanted kidney is relatively low in the first ten years after transplantation, exacerbation of a metabolic disorder with an associated risk of cardiovascular disease $[1,2]$. The recurrence of diabetic nephropathy is not considered a contraindication for kidney transplantation.

In our patient, terminal renal failure is caused by diabetic nephropathy. The method of choice in the treatment of renal replacement therapy (hemodialysis), was a kidney transplant. In young recipients with juvenile diabetes (type 1 diabetes), simultaneous kidney and pancreas transplantation is the first-choice method that can significantly improve quality and prolong life. Diabetes is a serious illness that affects the outcome of the transplant, as well as the morbidity and mortality, primarily because of the increased cardiovascular and infectious risk. Because the incidence of diabetic 
nephropathy after renal transplantation is significant, strict control of diabetes and hypertension is recommended, with the utilization of Angiotensin-converting enzyme inhibitors (ACEI) and/or angiotensin receptor blocers (ARB) to prevent or mitigate this risk [3-5].

Posttransplant complications significantly affect morbidity and mortality. Patients after kidney transplantation must take immunosuppressive drugs to prevent rejection of the transplanted organ. The most commonly used triple immunosuppressive therapy: calcineurin inhibitor (cyclosporine/ tacrolimus), antiproliferative drugs (mycophenolate mofetil or azathioprine) and corticosteroids. Inhibitors of calcineurin have a well-known toxic effect on the kidney, but less is known about their potent neurotoxic effect. Cyclosporine can be neurotoxic (up to $50 \%$ of transplant patients). The range of neurological disorders caused by calcineurin inhibitors ranges from very mild symptoms to severe changes with potentially catastrophic consequences. The development of neurological side effects of calcineurin inhibitors benefit from: high drug concentration, hypomagnesaemia, high doses of corticosteroids, hypertension, and infection [2,6]. The changes are usually reversible and reversed upon discontinuation of the drug, with fewer patients having lasting effects, regardless of the change or discontinuation of immunosuppressive therapy as was the case in our patient.

Tuberculosis (TB) is not a rare disease after kidney transplantation, and can be life-threatening. TB treatment in patients with a kidney transplant must be performed in the same way as in the general population. This means that treatment with a combination of four drugs - rifampin, isoniazid, ethambutol and pyrazinamide, must be followed for 2 months, followed by treatment with a combination of isoniazid and rifampin for 4 months. Ethambutol should not be given initially if the isoniazid resistance rate in the population is less than $4 \%$. Because rifampin decreases plasma concentrations of calcineurin antagonists and rapamycin, the levels of these agents in the blood should be closely monitored. Rifabutin may be prescribed as an alternative to rifampin because this drug is less effective in inducing the microsomal enzymes P450. Kidney transplant candidates and renal graft recipients should be screened for latent TB infection.

Latent TB infection is best treated with a daily dose of $300 \mathrm{mg}$ of isoniazid for 9 months [7,8]. Central nervous system (CNS) infections usually occur during the first year after transplantation, and are manifested by disorders of mental status, headache, fever, and focal neurological outbreaks. Unclear symptomatology often causes delay in accurate diagnosis. Clinically, CNS infections can manifest as meningitis, progressive dementia, and focal infections $[9,10]$. In addition to immunosuppressive therapy, the tendency for post-transplant infections also contributes to the decreased immune response of patients in uremia, anemia and impaired blood coagulation [11]. Specific meningoencephalitis is a rare complication and is undiagnosed frequently, and can leave severe neurological sequelae, such as in the present patient: hemiparesis, right cervical neural facial paresis, motor aphasia, and impaired hearing. For the diagnosis of specific meningoencephalitis in this case, there were positive medical history, clinical findings, laboratory findings, CSF and brain CT.

\section{Conclusion}

Prognosis in immunocompromised patients with CNS infection is poor. Only timely and prompt diagnosis can improve the patient's survival rate. It is recommended that prophylactic administration of isoniazid tuberculostatics at a dose of $300 \mathrm{mg}$ for 9 months should be included in the therapeutic protocol after renal transplantation.

\section{References}

1. Cohen J, Hopkin J, Kurtz J. Infectious complications after renal transplantation. In: Morris PJ (ed). Kidney Transplantation; Principles and Practice. WB Saunders Company, Philadelphia, London, New York, Toronto. 2002; 468-490

2. Živčić-Ćosić S, Trobonjača Z, Rački S. Imunosupresivno liječenje kod presađivanja bubrega. Medicina. 2010; 4: 413-23.

3. Campbell PM. Pathology of acute rejection in the renal allograft. ASHI quaterly. Third quarter. New Jersey. ASHI. 2004: 86.

4. Ležaić $\mathrm{V}$, Radivojević $\mathrm{D}$, Blagojević R, Đukanović LJ. Infection of central nervous system after kidney transplantation. Transplant Infect Dis. 2002; 4: 167-168.

5. Kidney Disease. Improving Global Outcomes (KDIGO) Transplant Work Group. KDIGO clinical practice guideline for the care of kidney transplant recipients. Am J Transplant. 2009; 9 (Suppl 3): S1-155. PubMed: https://pubmed.ncbi.nlm.nih.gov/19845597/

6. Brennan DC, Bohl D. Infectious complications in renal transplant recipients. In: Malluche HH, Sawaya BP, Hakim RM, Sayegh MH (eds). Clinical nephrology, dialysis and transplantation. 2004; 1-34.

7. Randhawa $P$, Brennan $D C$. BK virus infection in transplant recipients: An overview and update. Am J Transplant. 2006; 6: 2000-2005. PubMed: https://pubmed.ncbi.nlm.nih.gov/16771813

8. Ležaić $\mathrm{V}$, Radivojević $\mathrm{R}$, Radosavljević $\mathrm{R}$, Blagojević $\mathrm{R}$, Simić $\mathrm{S}$, et al. Does tuberculosis after kidney transplantation follows the trend of tuberculosis in general population? Renal Failure. 2001; 23: 97-106. PubMed: https://www.ncbi.nlm.nih.gov/pubmed/11256535

9. Singh N, Paterson DL. Mycobacterium tuberculosis infection in solid organ transplant recipients: impact and implications for menagement. Clin Infect Dis. 2008; 27: 1266-1277.

10. Morath C, Schmied B, Mehrabi A, Weitz J, Schmidt J, et al Simultaneous pancreas-kidney transplantation in type 1 diabetes. Clin Transplant. 2009; 23 Suppl 21: 115-20.

PubMed: https://www.ncbi.nlm.nih.gov/pubmed/19930324

11. Joyce AT, lacoviello JM, Nag S. End-stage renal disease-associated managed care costs among patients with and without diabetes. Diabetes Care. 2004; 27: 2829-2835.

PubMed: https://www.ncbi.nlm.nih.gov/pubmed/15562193 